\title{
O que Explica o Sucesso de Jogos no Ensino de Engenharia de Software? Uma Teoria de Motivação
}

\author{
Márcia Souza, César França \\ Mestrado Profissional em Engenharia de Software \\ CESAR.EDU - Recife - PE - Brazil \\ Universidade Federal do Rural de Pernambuco (UFRPE) \\ Recife - PE - Brazil \\ marciavr.souza@gmail.com, cesar@franssa.com
}

\begin{abstract}
The development of games for teaching software engineering disciplines has proven to be a quite successful instructional alternative. It is known, however, that the attractiveness of the games is highly associated with sophisticated social and psychological mechanisms. This article presents a systematic review conducted in 2008 WEI Annals to 2015, which shows that there is a significant convergence between the features of educational games in this literature and the Theory of Motivation and Satisfaction of Software Engineers. Thus, this theory can serve as a basic mechanism for the conceptual design of even more attractive educational games in this area.
\end{abstract}

Resumo. $O$ desenvolvimento de Jogos para ensino das disciplinas de engenharia de software tem se mostrado como uma alternativa instrucional de bastante sucesso. É sabido, no entanto, que a atratividade dos jogos está associada a mecanismos sociais e psicológicos altamente sofisticados. Neste artigo, apresentamos uma revisão sistemática conduzida nos anais do WEI de 2008 a 2015, que demonstra que há uma convergência significativa entre as características dos jogos educacionais propostos nesta literatura e os pressupostos da Teoria de Motivação e Satisfação de Engenheiros de Software. Isto posto, esta teoria poderá servir como mecanismo de base para o design conceitual de jogos educacionais ainda mais atrativos nesta área.

\section{Introdução}

Para garantir a formação de um bom profissional na área tecnológica, bem como um engenheiro de software, é preciso que durante a graduação ele tenha uma boa base de conhecimentos básicos, desde programação até processos de desenvolvimento de software. A finalidade é que futuramente no mercado ele possa ser capaz de atuar profissionalmente desenvolvendo com qualidade produtos de softwares.

Os professores, das disciplinas dos cursos na área de tecnologia, são desafiados constantemente a instigar os estudantes para que eles possam prosseguir de maneira positiva no curso, dando-lhes uma boa base de conhecimento aliada a motivação, onde segundo Silva et al. (2011) pode estar relacionado à carência de práticas educacionais alternativas às tradicionais. Os alunos esperam dos professores não apenas a teoria, 
porém o maior desafio é na utilização de uma metodologia na sala de aula que possa favorecer um maior número de alunos e consequentemente o professor.

Uma das propostas de melhoria do aprendizado em sala de aula são os jogos educacionais. $\mathrm{Na}$ literatura encontram-se vários trabalhos que demonstram profissionais de educação utilizando-os como ferramenta de auxílio ao aprendizado. Dentre as vantagens de utilizar jogos educacionais, podemos destacar a motivação e o aprendizado vivencial. Os jogos permitem que os alunos possam vivenciar experiências teóricas e práticas. No entanto, trabalhos recentes têm apontado que a atratividade dos jogos advém fundamentalmente da sua capacidade de simulação de fatores psicossociais relevantes para a motivação humana [Hamari, 2013][Chou, 2014].

Nesta pesquisa, nos propusemos então a revisar a literatura sobre jogos educacionais das disciplinas dos cursos de computação, e avaliar se a presença/ausência dos fatores previstos pela Teoria de Motivação e Satisfação de Engenheiros de Software (TMS-ES) por França (2014) estão associados ao sucesso reportado do jogo. A TMS-SE é um trabalho que envolve as principais teorias de motivação do campo da psicologia desenvolvidas no último século adaptando-os para a realidade específica do trabalho na engenharia de software. A TMS-SE foi desenvolvida no campo do comportamento organizacional, mas há razões para acreditar que ela possa ter um impacto significativo na área da educação [Richter, 2014]. Neste artigo, apresentamos uma análise preliminar resultantes da literatura específica do Workshop de Educação em Computação (WEI) sob este conjunto de características da TMS-SE.

Este artigo está organizado da seguinte forma: será explicado sobre a importância da engenharia de software e dos jogos educacionais e serão abordados os elementos motivacionais que servirão de base para a condução desta pesquisa. $\mathrm{Na}$ terceira seção será explicada a metodologia que foi conduzida para este trabalho. $\mathrm{Na}$ quarta seção serão exibidos os resultados desta pesquisa e as discussões dos resultados. Por fim, na quinta seção, serão explicadas a conclusão desta pesquisa.

\section{Referencial Teórico}

\subsection{Desafios no ensino da Engenharia de Software}

Engenharia de software é uma das áreas mais importantes da Tecnologia da Informação, sendo especialmente central para a formação adequada de Engenheiros de Software [Sommerville 2011]. Tem como objetivo apoiar o desenvolvimento profissional de software, cobrindo todos os aspectos da produção de um software. Monsalve et al. (2010), comentam que a engenharia de software é uma área com aspectos teóricos e práticos, tornando fundamentais as experiências e decisões da prática no desenvolvimento de softwares de qualidade, com economia, utilidade e no prazo esperado. Porém, Benitti e Molléri (2008) concordam que a engenharia de software é uma área muito jovem e sofre contínuas mudanças nos seus fundamentos tecnológicos concretizadas nos métodos e ferramentas de suporte, portanto necessita de métodos de ensino lúdicos e dinâmicos que possam contribuir na aprendizagem do estudante.

Contudo, a forma como algumas disciplinas de engenharia de software comumente são ensinadas para os alunos não permite que a prática seja trabalhada podendo gerar insatisfação e consequentemente profissionais despreparados com as 
técnicas utilizadas no mercado de trabalho. Andrade et al. (2008) em seu trabalho reforça a necessidade da relação pedagógica entre professor e aluno em uma estrutura metodológica e planejada com teorias modernas de aprendizagem. Como a finalidade na sala de aula é estimular ideias dos alunos, ensinar apenas com aulas expositivas tradicionais pode dificultar o aprendizado.

Uma das propostas metodológicas para ensino de engenharia de software e suas disciplinas, são os jogos educacionais. Sabe-se que os jogos educacionais, segundo Nunes e Parreira (2015), têm sido intensamente utilizados por profissionais da área de educação como auxílio para a construção do conhecimento. Em sua pesquisa, Fukusawa et al. (2015) apontam alguns dos benefícios que os jogos educacionais podem trazer ao processo de ensino e aprendizagem como, por exemplo, a motivação e o aprendizado por descoberta. Portanto, os jogos podem proporcionar a vivência em experiências de aprendizagem concretas [Monsalve et al. 2010].

\subsection{Jogos para auxílio nas disciplinas de Engenharia de Software}

Na literatura é possível encontrar inumeros trabalhos utilizando jogos como auxilio ao aprendizado nas disciplinas dos cursos de engenharia de software. Os autores Rangel et al. (2015) observaram que utilizar jogos na educação proporcionam aos estudantes e profissionais um ambiente interativo e dinâmico provocando a motivação para avançar em um enredo, por meio de simulações e situações relacionadas ao tema abordado. Silva et al. (2015) comentam que uma abordagem alternativa às aulas tradicionais, devido elas serem mais teóricas e expositivas, é a utilização dos jogos, pois esta abordagem preza por uma teoria de motivação humana como ponto de partida.

Pode-se observar que para alcançar algum sucesso no que é proposto, além de atender aos objetivos educacionais, os jogos devem principalmente transmitir motivação e satisfação aos estudantes. Segundo Vahldick et al. (2015), se estes objetivos não forem alcançados, o jogador ficará aborrecido, desmotivado e desconcentrado, não atingindo os objetivos da aprendizagem. No entanto, a motivação é um elemento quase que acidental resultante do processo de design de jogos function-focused, uma vez que fatores motivacionais não são necessariamente conhecidos ou levados em conta no início deste processo [Chou, 2014]. Chou (2014) aponta que a abordagem humanfocused é capaz de garantir o sucesso do jogo no aspecto motivacional, pois esta abordagem adora uma teoria de motivação humana como ponto de partida.

\subsection{Teoria da Motivação e Satisfação dos Engenheiros de Software}

De acordo com França (2014), o sucesso dos projetos de software depende do gerenciamento apropriado da motivação e satisfação no trabalho. $\mathrm{O}$ estudo realizado pelo autor, investigou um conjunto de características presentes no ambiente de tabalho dos engenheiros de software que podem influenciar positivamente ou negativamente seu desempenho. Relevantes teorias advindas do campo da psicologia, tais como a Job Satisfaction Theory por Locke (1969) e Job Characteristics Theory por Hackman (1980) foram então combinadas e adaptadas a uma nova teoria explanatória da motivação e satisfação no trabalho de engenheiros de software (TMS-SE), a qual tem como objetivo esclarecer as características do trabalho que influenciam a motivação dos engenheiros de software. 
Segundo esta teoria, a motivação atua como um elemento moderador na transformação do desempenho potencial de um engenheiro, em seu desempenho real. De forma concreta, a motivação do engenheiro é representada por uma combinação de engajamento e concentração na execução de uma tarefa, e as características específicas da tarefa, como percebidas pelos engenheiros de software, condicionam este comportamento. As características influentes seriam então: a utilidade do conhecimento gerado, a demanda por criatividade, o impacto social, a variedade da tarefa, uma definição clara do que é esperado, a autoconfiança técnica e o engajamento de seus pares. Por se tratar de uma atividade fundamentalmente intelectual, a sobrecarga cognitiva atuaria de forma negativa sobre a motivação destes profissionais.

A satisfação, por sua vez, é representada concretamente pela felicidade resultante do julgamento do engenheiro sobre os resultados atingidos pelo seu desempenho dadas as condições conjunturais (físicas, organizacionais e sociais) nas quais a tarefa foi conduzida. O principal resultado da satisfação é o reforço (positivo ou negativo) sobre o desempenho potencial do engenheiro de software para execução de outras tarefas no futuro.

O uso desta teoria como fundamento para o design de jogos educacionais poderia ser útil, uma vez que a teoria permite orientações, como por exemplo:

(i) A TMS-ES afirma que a forma mais simples de melhorar a confiança técnica de um engenheiro de software é proporcionando oportunidades de experimentar e aprender novas tecnologias, ferramentas e métodos antes de adotá-las. A partir desta teoria, os jogos que constroem a autoconfiança técnica dos alunos tem mais chance de serem úteis em sala de aula.

(ii) Com a tecnologia em constante evolução, França (2014) observou que os engenheiros de software necessitam de aprendizado constante e a utilidade deste aprendizado podem refletir na qualidade da sua produção, desempenho, ou até mesmo aumentar a sua oportunidade de encontrar outros empregos. $\mathrm{O}$ aluno, na sala de aula, ao perceber a utilidade daquilo que está aprendendo, pode refletir também no seu desempenho.

(iii)A criatividade, segundo a TMS-ES, é uma característica intrínseca do processo produtivo dos engenheiros de software e é composto por uma série de atividades criativas ou não. Há uma maior concentração na parte criativa quando se refere a suas tarefas favoritas e tendem a falar quando não gostam das tarefas. $\mathrm{O}$ desafio seria manter a motivação na execução de parte que não são criativas. Na sala de aula, estimular a criatividade do aluno é um grande desafio e dependendo do jogo, é possível estimular a criatividade do aluno para certas tarefas.

\section{Metodologia}

Foram pesquisados nos Anais da base WEI e selecionados os artigos que tivessem trabalhos com jogos que auxiliassem no aprendizado de ensino superior das disciplinas de Engenharia de Software, preferencialmente os que validassem com alunos. Ficaram de fora os artigos que utilizaram o Scratch, ainda que o produto final fosse um jogo, é considerado um ambiente de programação fácil que não requer conhecimentos prévios 
de algoritmos ou de programação para o seu manuseio [Cristovão, 2008]. O período das publicações foi entre 2008 a 2015 nos anais disponíveis de forma eletrônica na internet ${ }^{1}$.

O propósito dos jogos estudados neste trabalho estão compreendidos entre as disciplinas básicas para todos os cursos e as específicas do curso de Engenharia de Software. Portanto os objetivos educacionais dos jogos selecionados deste trabalho fazem parte das Diretrizes Curriculares (2003) dos cursos Engenharia de Software.

Foram incluídos na pesquisa os artigos que apresentavam no conteúdo um relato da experiência do uso de algum jogo educacional com os alunos dos cursos de engenharia de software. Inclusive os que não tomavam como base uma validação necessariamente experimental e formal. Consideramos igualmente os jogos independentemente da plataforma digital ou analógico. Descartamos os trabalhos com alunos do ensino médio e fundamental e incluímos dois trabalhos do curso técnico, sendo um deles para ensino à distância. A seleção inicial considerou os artigos que explicitavam no título ou no resumo que se tratavam de ferramentas em geral que apoiassem o ensino de disciplinas de engenharia de software. Em um segundo momento, foi efetuada uma prévia leitura da metodologia dos trabalhos para averiguar se eram jogos. Artigos sobre ferramentas de apoio à execução de tarefas e/ou processos que não se tratavam de jogos foram então removidos. A lista final dos 20 trabalhos selecionados pode ser encontrada no Apêndice deste.

Estes resultados ainda são passíveis de falhas, uma vez que as avaliações dos artigos foram conduzidas por apenas um pesquisador, mesmo que com o apoio do seu orientador. Representando, portanto, uma ameaça à validade deste trabalho.

Apoiado na TMS-SE, verificamos a presença ou ausência dos fatores motivacionais em cada um dos jogos relatados nos trabalhos, com uma leitura mais aprofundada na metodologia, resultados e discussões. Foi construído então uma tabela com os artigos e em cada uma das características apresentadas acima, com exceção do objeto de aprendizagem, colocamos os valores SIM para os trabalhos que confirmassem a característica, NÃO para os que não confirmassem a características e '?' para os que não apresentaram o resultado para a característica. Conservamos nesta pesquisa os trabalhos [11] e [20], apesar de utilizarem a mesma ferramenta, CyberCIEGE, as abordagens na sala de aula foram diferentes. Nesta tabela, também anotamos os objetivos da aprendizagem, referindo-se ao conteúdo da disciplina que o jogo se propõe a auxiliar. Sendo assim, os elementos analisados foram:

a. Objeto da aprendizagem. Apenas os trabalhos que tratassem de jogos voltados para o ensino de disciplinas dos cursos de engenharia de software;

\section{Elementos Sociais e Individuais}

b. Engajamento dos colegas;

No que diz respeito ao engajamento, segundo a TMS-SE um engenheiro motivado é um engenheiro engajado, conforme observado no jogo SE-RPG [1], em seu trabalho, o jogo foi executado em equipe contribuindo para engajamento entre os colegas. Já no trabalho [8], o iTestLearning é um jogo single-player

\footnotetext{
${ }^{1} \mathrm{http}: / /$ www.lbd.dcc.ufmg.br/colecoes/wei/
} 
(jogo para somente um jogador) onde é realizado um planejamento de teste de software a partir da especificação de projeto.

c. Construção da autoconfiança técnica do aluno;

Sobre a autoconfiança técnica do aluno, [2] em seu trabalho constatou que foi através do jogo que os alunos puderam relembrar e ressignificar conteúdos importantes para as disciplinas sequintes contribuindo para a construção da autoconfiança dos alunos. Já no trabalho de [5], constataram nas avaliações que o jogo não fez diferença em relação a aprendizagem quando comparado com uma aula prática.

\section{Características da Tarefa}

d. O aluno compreende o impacto social daquilo que está aprendendo;

Em relação a compreender o impacto social do que está aprendendo, [5] relataram que os alunos acreditaram que o jogo aliado com as aulas práticas os ajudaram a aprender o conteúdo. Nos demais trabalhos esta característica não estava declarada.

e. O aluno percebe a utilidade daquilo que está aprendendo;

No que diz respeito à percepção da utilidade daquilo que está aprendendo, os alunos deste trabalho [10] perceberam a utilidade do ambiente de investimentos entre equipes que o jogo proporcionou. Já [13] apontaram alguns problemas na adoção do jogo, como por exemplo a desistência de alguns aluno no desafio proposto.

\section{f. Trata-se de uma quebra de rotina;}

Considerando que a ferramenta de auxílio ao aprendizado de algumas disciplinas de engenharia de software trata-se de um jogo, pode-se afirmar que é uma quebra de rotina às aulas tradicionais. Todos os artigos concordaram que o jogo tratavase de uma quebra de rotina. Em seu trabalho, [18] comentaram que os discentes se mostraram receptivos às inovações e explicaram que esse tipo de iniciativa é louvável frente a disciplinas teóricas, que demandam o estudo de conteúdos e que, em aulas expositivas, é feito de modo cansativo.

g. Explora a criatividade do aluno;

Dentre os jogos que exploram a criatividade do aluno, [15] afirmaram que uma das vantagens experimentais está a possibilidade de simular os cenários criados pelo professor, contribuindo para aguçar a criatividade. A maioria dos trabalhos apressentaram jogos com estratégias que exploram a criatividade do aluno. Poucos trabalhos não deixaram explícito no texto esta característica.

h. Possui tarefas bem definidas;

Com relação à definição das tarefas, [12] dividiu seu jogo em duas fases em que o aluno deverá jogar 16 missões e verificou a imersão dos alunos e com o conteúdo dentro dos objetivos da aprendizagem.

\section{Características Ambientais}

i. Possui a carga cognitiva desbalanceada negativamente. 
No trabalho [09], quando perguntado aos estudantes se utilizar o jogo simultaneamente ao estudo da disciplina facilitaria o processo de ensinoaprendizagem da disciplina, $100 \%$ responderam positivamente e alegaram que o jogo teria ajudado bastante principalmente nos seus estudos para realização das avaliações. Todos os trabalhos apresentaram carga cognitiva balanceada, com desafios relacionados ao conteúdo de sala de aula.

Com essas informações podemos avaliar se estes elementos estão presentes nos trabalhos selecionados e principalmente se o sucesso do uso desses jogos estão relacionados com a presença ou não desse conjunto de elementos.

\section{Resultados e Discussão}

Obsevou-se que de 2008 a 2011, poucos trabalhos envolveram jogos e que tivessem validação. Nenhum trabalho do ano de 2009 foi selecionado. A partir do ano de 2013 houve muito mais trabalhos com os critérios de pesquisa que especificamos no capítulo anterior. Na Tabela 1, com relação aos objetos de aprendizagem, entre os artigos selecionados observamos um número maior de jogos de aprendizagem de algoritmos. Em seguida estão os jogos voltados para auxílio de programação. Na tabela 2, encontram-se as respostas da avaliação de cada artigo (de 1 à 20), para cada fator motivacional (de $b$ à $i$ ). No Apêndice estão numerados os trabalhos selecionados para esta pesquisa e para as análises utilizaremos os índices das características de A a I descritos no capítulo anterior.

Tabela 1. Análise dos artigos sobre o Objeto da Aprendizagem

\begin{tabular}{|ll|ll|}
\hline \multicolumn{1}{|c|}{ Objeto da Aprendizagem } & Ref.* & \multicolumn{1}{|c|}{ Objeto da Aprendizagem } & Ref.* \\
\hline Processo de desenvolvimento de & {$[1]$} & Segurança em Redes de & {$[11$ e 20] } \\
software & {$[1,15]$} & Computadores & {$[9]$} \\
Gestão de Projetos & {$[2,3$ e 4$]$} & Empreendedorismo & {$[10]$} \\
Algoritmos & {$[3,14]$} & Lógica de Programação & {$[12]$} \\
Estrutura de Dados & {$[5]$} & Teste de Software & {$[8]$} \\
Engenharia de Requisitos & {$[7$ e 19] } & Circuitos Digitais & {$[13]$} \\
Programação & {$[6,17]$} & Métodos de Ordenação & {$[16]$} \\
Programação Orientada a Objetos & Sistema da Informação & {$[18]$} \\
Tipos de Variáveis & {$[7]$} & \multicolumn{2}{c}{} \\
\hline
\end{tabular}

*artigos referenciados no Apendice A

Nesta pesquisa, encontramos 9 dos 20 trabalhos que afirmaram ter observado o aumento da autoconfiança técnica dos alunos. Foram observados 19 trabalhos em que a prática dos jogos não provocou a sobrecarga cognitiva dos alunos. Apenas um trabalho não continha essa informação explícita.

Observamos que dos 20 artigos, 8 confirmaram a presença da percepção do impacto social do que estavam aprendendo. Na pesquisa, 10 trabalhos afirmam que os alunos percebem a utilidade do que estão aprendendo e 18 trabalhos permitem explorar a criatividade dos alunos na sala de aula. 19 artigos apresentaram jogos com tarefas bem definidas.

Tabela 2. Análise dos Fatores Motivacionais

\begin{tabular}{|l|llllllllllllllllllllllll}
\hline $\begin{array}{l}\text { Artigo } \\
\text { / Fator }\end{array}$ & 1 & 2 & 3 & 4 & 5 & 6 & 7 & 8 & 9 & 10 & 11 & 12 & 13 & 14 & 15 & 16 & 17 & 18 & 19 & 20 & Total \\
\hline
\end{tabular}




\begin{tabular}{|c|c|c|c|c|c|c|c|c|c|c|c|c|c|c|c|c|c|c|c|c|c|}
\hline$b$. & $\checkmark$ & $\checkmark$ & $\checkmark$ & $?$ & $\checkmark$ & $\checkmark$ & $\checkmark$ & $x$ & $x$ & $\checkmark$ & $x$ & $x$ & $x$ & $x$ & $x$ & $\checkmark$ & $\checkmark$ & $\checkmark$ & $\checkmark$ & $\checkmark$ & $12 / 20$ \\
\hline$c$. & $\checkmark$ & $\checkmark$ & $x$ & $?$ & $x$ & $?$ & $?$ & $\checkmark$ & $\checkmark$ & $\checkmark$ & $?$ & ? & $?$ & $\checkmark$ & $\checkmark$ & $?$ & $?$ & $?$ & $\checkmark$ & $\checkmark$ & $9 / 20$ \\
\hline$d$ & $\checkmark$ & $\checkmark$ & $?$ & $?$ & $\checkmark$ & $?$ & $?$ & $\checkmark$ & $?$ & $\checkmark$ & $?$ & ? & $?$ & $\checkmark$ & ? & $\checkmark$ & $?$ & $?$ & $?$ & $\checkmark$ & $8 / 20$ \\
\hline$e$ & $\checkmark$ & $\checkmark$ & $?$ & $?$ & $?$ & $?$ & $?$ & $\checkmark$ & $?$ & $\checkmark$ & $\checkmark$ & ? & $x$ & $\checkmark$ & $\checkmark$ & $?$ & $?$ & $\checkmark$ & $\checkmark$ & $\checkmark$ & $10 / 20$ \\
\hline$f$. & $\checkmark$ & $\checkmark$ & $\checkmark$ & $\checkmark$ & $\checkmark$ & $\checkmark$ & $\checkmark$ & $\checkmark$ & $\checkmark$ & $\checkmark$ & $\checkmark$ & $\checkmark$ & $\checkmark$ & $\checkmark$ & $\checkmark$ & $\checkmark$ & $\checkmark$ & $\checkmark$ & $\checkmark$ & $\checkmark$ & $20 / 20$ \\
\hline$g$ & $\checkmark$ & $\checkmark$ & $\checkmark$ & $\checkmark$ & $\checkmark$ & $\checkmark$ & $\checkmark$ & $\checkmark$ & $?$ & $\checkmark$ & $\checkmark$ & $\checkmark$ & $\checkmark$ & $\checkmark$ & $\checkmark$ & $\checkmark$ & $?$ & $\checkmark$ & $\checkmark$ & $\checkmark$ & $18 / 20$ \\
\hline$h$. & $\checkmark$ & $\checkmark$ & $x$ & $\checkmark$ & $\checkmark$ & $\checkmark$ & $\checkmark$ & $\checkmark$ & $\checkmark$ & $\checkmark$ & $\checkmark$ & $\checkmark$ & $\checkmark$ & $\checkmark$ & $\checkmark$ & $\checkmark$ & $\checkmark$ & $\checkmark$ & $\checkmark$ & $\checkmark$ & $19 / 20$ \\
\hline$i$ & $x$ & $x$ & $?$ & $x$ & $x$ & $x$ & $x$ & $x$ & $x$ & $x$ & $x$ & $x$ & $x$ & $x$ & $x$ & $x$ & $x$ & $x$ & $x$ & $x$ & $0 / 20$ \\
\hline
\end{tabular}

$\checkmark$ - SIM, $x$ - NÃO, ? - Não foi possível inferir

Todas as características descritas são elementos importantes que podem ser utilizados para avaliação de jogos que apoiam o ensino de disciplinas de engenharia de software. Todos os jogos tiveram sucesso em algum aspecto e a maioria dos trabalhos apontaram as falhas e se propuseram a corrigir e/ou repetir o experimento com os alunos. A partir dessas informações podemos afirmar que estes elementos propostos por França (2014) em sua teoria TMS-SE estão largamente presentes nos trabalhos selecionados.

\section{Conclusão}

Este trabalho efetuou uma avaliação dos jogos para auxílio de aprendizagem de disciplinas de engenharia de software, publicado nos anais do WEI entre os anos de 2008 a 2015, sob o conjunto de características propostas na teoria de motivação e satisfação dos engenheiros de software. Estes conjuntos de características que estão presentes pelo menos parcialmente em todos os jogos pesquisados, podem atestar que uma das principais preocupações ao desenvolver um jogo é se provocará a motivação e a satisfação dos alunos.

É importante notar que este trabalho é um esforço preliminar no sentido de revelar os aspectos motivacionais relevantes para o design de jogos educacionais nas disciplinas de engenharia de software. Como forma de continuar este trabalho, planejamos conduzir uma revisão sistemática mais ampla, expandindo a base de artigos para incluir outras conferência e jornais relevantes nacionais e internacionais, e observar as evidências reportadas sobre a experiência de aprendizado, buscando identificar a sua relação com a TMS-ES. Em seguida, pretendemos avaliar o uso da teoria em um processo real de concepção de um jogo educacional na área de engenharia de software, bem como os efeitos desse uso no potencial motivador do jogo nos alunos.

\section{Referências}

Andrade, R. M. de C., Marinho, F. G., Leitão, V. L. e Rocha, L. S. (2008). Uma Proposta de Metodologia para o Ensino de Engenharia de Software. Fórum de Educação em Engenharia de Software, n. Mdcc.

Benitti, F. e Molléri, J. (2008). Utilização de um RPG no ensino de gerenciamento e processo de desenvolvimento de software. WEI - Workshop sobre Educação em Computação. (pp. 358-267).

Chou, Yu-kai (2014) Actionable Gamification - Beyond Points, Badges, and Leaderboards. Leanpub. 
Cristovão, H. M. (2008). Aprendizagem de Algoritmos num Contexto Significativo e Motivador: Um Relato de Experiência. WEI - Workshop sobre Educação em Computação. (pp.30).

Diretrizes Curriculares Conselho Nacional de Educação e Câmara de Educação Superior (2003). Ministério da Educação. http://www.sbc.org.br/documentos-da-sbc/send/131curriculos-de-referencia/761-diretrizes-curriculares-consulta-publica. Maio, 2016.

França, C. (2014). A Theory of Motivation and Satisfaction of Software Engineers. p. 200.

Fukusawa, J., Carniello, A. e Carniello, A. (2015). JOGOS DIGITAIS NO ENSINO E APRENDIZAGEM DE ENGENHARIA. Gestão Universitária, v. 1, p. 1-8.

HACKMAN, J. R. Work redesign and motivation. Professional Psychology, v. 11, n. 3 , p. $445-455,1980$.

Hamari, J., \& Koivisto, J. (2013). Social motivations to use gamification: an empirical study of gamifying exercise. Proceedings of the 21st European Conference on Information Systems, Utrecht, Netherlands, June 5-8, 2013.

LOCKE, E. A. What is job satisfaction? Organizational Behavior and Human Performance, p. 309-336, 1969.

Monsalve, E. S., Werneck, V. M. B. e Cesar, J. (2010). SimulES-W : Um Jogo para o Ensino de Engenharia de Software. Anais do III Fórum de Educação em Engenharia de Software, p. 17-26.

Nunes, I. F. e Parreira, P. A. (2015). RPG4Sorting - Um Jogo Educacional para Auxílio ao Ensino de Métodos de Ordenação. XXIII Workshop sobre Educação em Informática.

Rangel, D., Leite, A. e Delfino, S. S. (2015). Gsprojects - ambiente para simulação da gestão de projetos de software. $23^{\circ}$ WEI - Workshop sobre Educação em Computação.

Richter, G., Raban, D., \& Rafaeli, S. (2014). Studying Gamification: The Effect of Rewards and Incentives on Motivation. Gamification In Education And Business, 21-46. doi:10.1007/978-3-319-10208-5 2.

Silva, R. J. M., Azevedo, W. L. V, Nascimento, R. P. e Paxiúba, C. M. C. (2015). MercadoSI : Um jogo de tabuleiro para o ensino de Sistemas de Informação. $23^{\circ}$ WEI Workshop sobre Educação em Computação.

Silva, T. G., Müller, F. M., e Bernardi, G. (2011). Panorama do Ensino de Engenharia de Software em Cursos de Graduação Focado em Teste de Software: Uma Proposta de Aprendizagem Baseada em Jogos. RENOTE, 9(2).

Sommerville, I. (2011). Engenharia de Software. Pearson/ Addison Wesley.Teixeira, M. R. W., Aguiar, G. D. F. e Ferlin, E. P. (2010). O Jogo Imagem \& Ação como Estratégia de Aprendizagem para o Conteúdo de Algoritmos Genéticos. WEI - XVIII Workshop sobre Educação em Computação, n. 843, p. 843-846.

Vahldick, A., Mendes, A. J. e Marcelino, M. J. (2015). Testando a Diversão em um Jogo Sério para o Aprendizado Introdutório de Programação. $23^{\circ}$ WEI - Workshop sobre Educação em Computação. 


\section{Apêncice}

[1] Benitti, F. B. V., \& Molléri, J. S. (2008) Utilização de um RPG no ensino de gerenciamento e processo de desenvolvimento de software.

[2] Teixeira, M. R. W., de França Aguiar, G., \& Pedro, E. (2010). O Jogo Imagem \& Ação como Estratégia de Aprendizagem para o Conteúdo de Algoritmos Genéticos.

[3] Digiampietri, L. A., Kropiwiec, D. D., \& Silva, R. A. (2010). O uso de jogos como fator motivacional em cursos de computação.

[4] Barbosa, L. S., Fernandes, T. C., \& Campos, A. M. (2011). Takkou: uma ferramenta proposta ao ensino de algoritmos.

[5] Silva, J. C., Sousa, S. P., Kulesza, R., \& Brito, A. V. (2012). Uma avaliação do emprego do jogo Modelando como apoio ao ensino de Engenharia de Requisitos.

[6] Oliveira, E. D., \& de Barros, R. M. (2013). ABstração Game Um Jogo Para Facilitar o Processo de Ensino e Aprendizagem da Orientação a Objetos.

[7] Kahwage, C., de França, E. L., Nunes, R. C., Carvalho, R., \& Souza, D. T. (2013). Jogo Baralho das Variáveis.

[8] Bezerra, C. I. M., \& Coutinho, E. (2013). Avaliação do Jogo iTestLearning: Um Jogo para o Ensino de Planejamento de Testes de Software.

[9] Leite, L., Sibaldo, M., Carvalho, T., Souza, R. (2014). Montanha de Chomsky: jogo tutor para auxílio no ensino de Teoria da Computação.

[10]Jucá, P. M., Alexandrino, A. O., Alexandrino, A. O., Rolim, G. F., \& de Almeida, C. D. A. (2014). Aplicação da Gamificação na Disciplina de Empreendedorismo.
[11]Herpich, F., Jardim, R. R., Silva, R. F., Voss, G. B., Nunes, F. B., Medina, R. D. (2014). Jogo Sério na Educação: Uma Abordagem para Ensino-Aprendizagem de Redes de Computadores (Fase II).

[12]Vahldick, A., Mendes, A. J., Marcelino, M. J., Hogenn, M., \& Schoeffel, P. (2015). Testando a Diversão em um Jogo Sério para o Aprendizado Introdutório de Programação.

[13]Cagnini, H. E., Charao, A. S., Patricia, P. D. A., \& de Azevedo, B. R. (2015). Mundo virtual Minecraft: uma Experiência no Ensino de Circuitos Digitais.

[14]Barbosa, W. A., Nunes, I. F., Inocencio, A. C., Oliveira, T. B., Parreira Júnior, P. A. (2015). DEG4Trees: Um Jogo Educacional Digital de Apoio ao Ensino de Estruturas de Dados.

[15]Leite, D. R. A., Delfino, S. S., Mélo, C. B., Medeiros, Á. F., \& de Andrade, H. G. R. (2015). GSPROJECTS-Ambiente para simulação da gestão de projetos de software.

[16]Freitas Nune, I., \& Júnior, P. A. P. (2015). RPG4Sorting-Um Jogo Educacional para Auxílio ao Ensino de Métodos de Ordenação.

[17]Silva Figueiredo, K., Ribeiro, J. M., Souza, R., \& Angelo, V. R. (2015). Uma Abordagem Gamificada para o Ensino de Programação Orientada a Objetos. XXXV Congresso da SBC. XXIII Workshop sobre Educação em Computação.

[18]Silva, R. J., Azevedo, W. L., Nascimento, R. P., \& Paxiúba, C. M. (2015). MercadoSI: Um jogo de tabuleiro para o ensino de Sistemas de Informação.

[19]Campos, A., Gardiman, R., \& Madeira, C. (2015). Uma ferramenta gamificada de apoio à disciplina introdutória de programação.

[20]Krassmann, A. L., Falcade, A., da Silva, L. E., \& Medina, R. D. (2015). Serious Games to Computer Networks Learning With CyberCIEGE: A Case Study in Brazilian Higher Education. 